\title{
Fiber Optic Sensors With Internal Referencing
}

Grigory Adamovsky

Lewis Research Center

Cleveland, Ohio

and

Duncan J. Maitland IV

Cleveland State University

Cleveland, Ohio

Prepared for the

Conference on "Optical Testing and Metrology II" sponsored by the Society of Photo-Optical Instrumentation Engineers Dearborn, Michigan, June 26-30, 1988

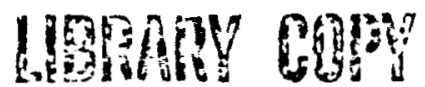 NOY 501988}


FIBER OPTIC SENSORS WITH INTERNAL REFERENCING

\author{
Grigory Adamovsky \\ National Aeronautics and Space Administration \\ Lewis Research Center \\ Cleveland, Ohio 44135
}

and

\author{
Duncan J. Maitland IV \\ Cleveland State University \\ Department of Engineering Technology \\ Cleveland, Ohio 44115
}

\begin{abstract}
SUMMARY
The main problem with amplitude modulating type sensors is that any variation in the intensity of the optical signal which occurs throughout the sensing system is interpreted by the photodetector as resulting from the sensor itself and is reflected as an error in the sensed parameter. To account for these errors, a referencing technique with the signal and reference channels separated in the time domain over the same fiber link can be used.

Selected sensing and signal processing techniques involving temporally separated signal and referencing channels are described. A transition from the time into the frequency domain is also discussed. Experimental data are presented.

\section{INTRODUCTION}

Amplitude modulating fiber optic sensors have found applications due to their simplicity and relatively low cost. These sensors respond to the sensed parameter by changing the intensity of the optical signal in the system. However a sensing system based on this principle is also susceptible to the intensity variations of the optical signal which occur throughout the system. These variations cannot be distinguished by a photodetector from intensity changes caused by the sensor itself and contribute to an error in the sensed parameter. Because of this the sensing system requires two channels, a reference and a signal, to compensate for variations in the source intensity, detector sensitivity, and transmission characteristics of the fiber-optic link (ref. 1).

Among different referencing techniques available, the one with channels separated in the time domain is attractive because it permits the use of the same fiber link for both channels. The technique is based on generating a pulse train using a pulse modulated light source and a fiber optic loop (refs. 2 and 3 ). The fiber optic loop with a sensor incorporated in it forms a sensor head. Thus, the separation of the channels occurs internally within the sensor head. To retrieve information about the measured parameter, the amplitudes of pulses in the train must be compared. The most recent technique developed to do this involves spectral analysis of the corresponding electrical signal from the photodetector (ref. 4). This transition into the frequency domain provides new opportunities for fiber optic sensors.
\end{abstract}




\section{TIME-TO-FREQUENCY CONVERSION}

The phenomenon of time-to-frequency conversion has been explained in reference 4. It can be performed by taking a Fourier transform of a periodic pulse train that exits a fiber optic loop. Analyses of different components of the frequency spectrum provide information about the relative amplitudes of the pulses in the train. The separation of these components is determined by the repetition rate of the initial pulse.

In the experimental setup shown in figure 1 a narrow repetitive pulse (pulse width at half power is $5 \mathrm{nsec}$ and repetition rate is $20 \mathrm{MHz}$ ) is sent into a reflective Fabry-Perot type fiber optic loop which consists of a $2.5 \mathrm{~m}$ long multimode fiber placed between two mirrors, Ml and $M 2$. The mirror $M 1$ is a semireflecting-semitransmitting mirror with a reflection coefficient of about 0.2 at a wavelength of $830 \mathrm{~nm}$. The mirror $M 2$ is a reflector positioned on $a$ translation stage $T$ to provide a longitudinal separation $\&$ between the fiber end and the reflective surface of the mirror. An optical system consisting of a collimating lens $L$ and a 50/50 cube beamsplitter BS provides the coupling of the light into the fiber loop and directs the returning pulse train towards the photodetector $D$ via the fiber $F$.

The configuration is chosen to provide secondary pulses due to reflection from the mirror M2 centered between periodic initial pulses. The amplitudes of the initial and the secondary pulses are adjusted to be approximately the same. Figure $2(a)$ depicts the shape of the optical signal that exits the fiber loop and figure $2(b)$ shows the frequency spectrum of this signal. If the entrance into the fiber optic loop is blocked, the secondary pulse would not be generated. This corresponds to a case when the mirror $M 2$ is moved away from the fiber end by such a distance that there is practically no light coupled back into the loop upon reflection. In this case the signal detected by the photodetector resembles the periodic initial pulses. Figures $3(a)$ and $3(b)$ show, respectively, the optical signal that reaches the photodetector when the entrance to the loop is blocked (the initial signal) and its frequency spectrum. It can be seen from the frequency spectra that the fundamental component at $20 \mathrm{MHz}$ for the initial signal ( $\mathrm{fig} .3(\mathrm{~b})$ ) is not present in the spectrum of the resultant signal that exits the loop (fig. 2(b)). At the same time the frequency component at $40 \mathrm{MHz}$ is present in both figures, being the fundamental of the resultant signal and the second harmonic of the initial one. If the amplitudes of the initial and the secondary pulses differ, the magnitude of the component at $20 \mathrm{MHz}$ would vary from zero for a case of equal amplitudes to a maximum for a case of an absent secondary pulse. The component at $40 \mathrm{MHz}$ can be used as a reference to compensate for intensity variations outside the fiber optic loop. This component also changes its magnitude with a change in the secondary pulse amplitude. However, the magnitude of the $40 \mathrm{MHz}$ component decreases with a decrease in the amplitude of the secondary pulse. It can be shown that a ratio of these magnitudes is independent of the initial pulse amplitude and is a function of the amplitude of the secondary pulse (ref. 4). Thus, a change in the amplitude of the secondary pulse can be detected by measuring and comparing the frequency components at $20 \mathrm{MHz}$ and $40 \mathrm{MHz}$.

One of the ways to change the amplitude of the secondary pulse is to change the amount of light coupled back into the fiber loop upon reflection from the mirror $M 2$. In this respect the technique could be used for displacement measurements. In order to do this the signal from the photodetector 
(See fig. 1) is split into two channels, each of which consists of an appropriate filter, an amplifier and a RF detector. The RF detector converts the filtered and amplified CW signal at either $20 \mathrm{MHz}$ or $40 \mathrm{MHz}$ into a DC output. The magnitude of the $D C$ output is a linear function of the magnitude of the corresponding frequency component. The DC signals emerging from the RF detectors have been measured and the ratio of these signals has been obtained for different distances between the loop and the mirror M2. The graph in figure 4 represents the experimentally obtained relationship between the ratio of the $D C$ signals $\mathrm{V}_{2} \mathrm{OMHz} / \mathrm{V}_{40 \mathrm{MHz}}$ and the mirror displacement $\ell$ over a range of displacements from 0 to $600 \mu \mathrm{m}$. During the experiment a sensitivity of $1 \mu \mathrm{m}$ has been observed.

\section{INITIAL DOUBLE PULSE TECHNIQUE}

The technique described in the above section of this article involves initial pulses with high repetition rate. The high repetition rate pulsing of laser diodes leads to overheating and affects the stability of the entire system. The disadvantages of this approach will have greater impact in sensing systems with shorter fiber optic loops and with correspondently higher repetition rate initial pulses. To minimize this problem a novel technique is proposed. The technique is based on using an initial double pulse with a lower repetition rate and sending this double pulse into the fiber optic loop described in the previous section. If the delay between the pulses in the initial double pulse is twice as long as the transit time for a pulse in the fiber optic loop, a train of four pulses results, equidistant in time.

In the experimental setup with $2.5 \mathrm{~m}$ long Fabry-Perot type fiber optic loop the transit time for a pulse in the $100 \mathrm{p}$ is about $25 \mathrm{nsec}$. The delay between pulses in the initial double pulse and the repetition rate are chosen to be about $50 \mathrm{nsec}$ and $1.82 \mathrm{MHz}$, respectively. Thus, the signal emerging from the loop consists of a periodic train of four pulses 25 nsec apart with the first and third being the initial pulses and the second and fourth being their respective secondary pulses. This signal and its spectrum, as well as the signal and its spectrum obtained when the entrance to the loop is blocked, are shown in figures 5 and 6 , respectively. The spectrum in this case consists of many components that appear under the frequency envelope of the Fourier transform due to the repetition rate. At the same time, two bands of frequency components centered at $20 \mathrm{MHz}$ and $40 \mathrm{MHz}$ can be easily recognized. It should be also noted that the behavior of these frequency bands is analogous to that of the corresponding frequency components at either $20 \mathrm{MHz}$ or $40 \mathrm{MHz}$ of the periodic initial pulses discussed above. The technique required to process the resultant train of four pulses in the frequency domain would also be analogous to that discussed above. The difference would be in using broad bandpass filters in each channel to capture several components in the corresponding band. However, this may lead to decrease in sensitivity.

\section{CONCLUSION}

Both configurations described in the paper are based on the use of a fiber optic loop to modulate the spectrum of an initial signal. It has been shown that high resolution can be achieved using high repetition rate pulses, a fiber optic loop of an appropriate length, and the time-to-frequency conversion.

However, high repetition rates may be avoided by using the initial double pulse technique presented. In this respect an increase in sensitivity could be achieved by increasing the number of pulses in the initial signal. 
Due to the fact that time-to-frequency conversion involves separation and processing of the two frequency bands by taking the ratio of the emerging signals, the result is independent of the intensity level of the initial signal. This makes the technique useful in propulsion control systems and other applications involving harsh environment.

\section{ACKNOWLEDGMENTS}

One of the authors (D.J. Maitland) wishes to acknowledge the support of the NASA Lewis Research Center (Grant NCC-3-58).

\section{REFERENCES}

1. G. Adamovsky, "Referencing in Fiber Optic Sensing Systems," in Optical Techniques for Sensing and Measurement in Hostile Environments, C.H. Gillespie and R.A. Greenwell, eds., Proc. SPIE 787, pp. 17-23 (1987).

2. W.B. Spillman, Jr. and J.R. Lord, "Self-Referencing Multiplexing Technique for Fiber-Optic Intensity Sensors," J. Lightwave Technol. LT-5, pp. 865-869 (1987).

3. G. Adamovsky, "Time Domain Referencing in Intensity Modulation Fiber Optic Sensing Systems," in Optical Testing and Metrology, C.P. Grover, ed., Proc. SPIE 661, pp. 145-151 (1986).

4. G. Adamovsky, "Amplitude Spectrum Modulation Technique for Analog Data Processing in Fiber Optic Sensing System with Temporal Separation of Channels," in Fiber Optic and Laser Sensors V, R.P. DePaula and E. Udd, eds., Proc. SPIE 838, pp. 264-270 (1987). 


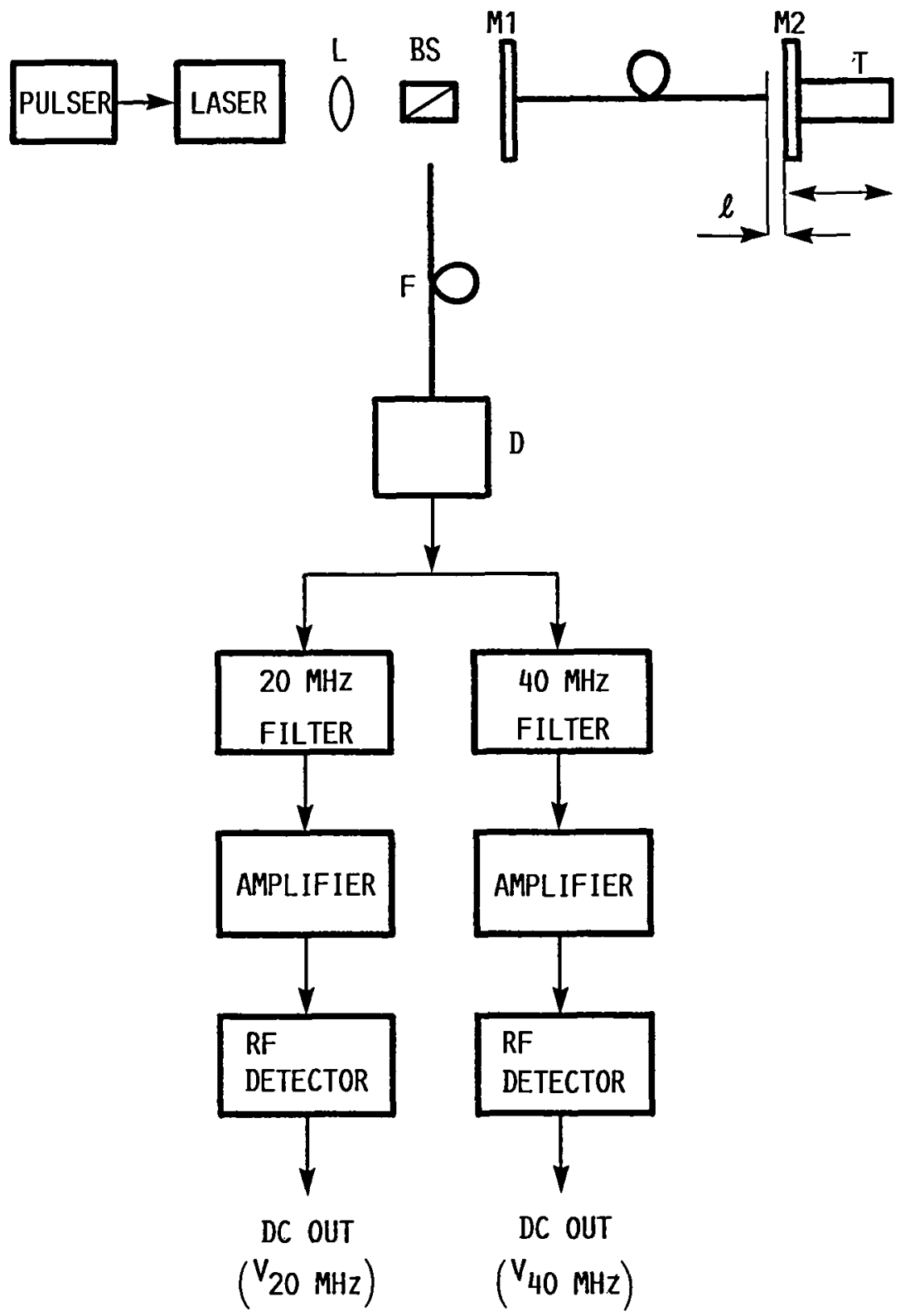

FIGURE 1.- EXPERIMENTAL SETUP. 


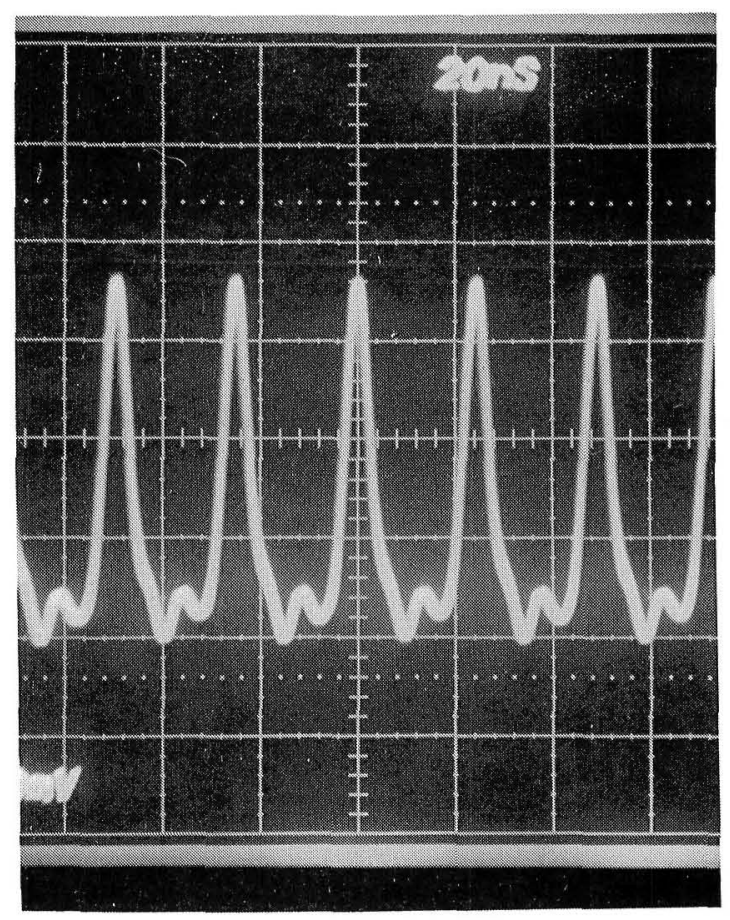

(a)
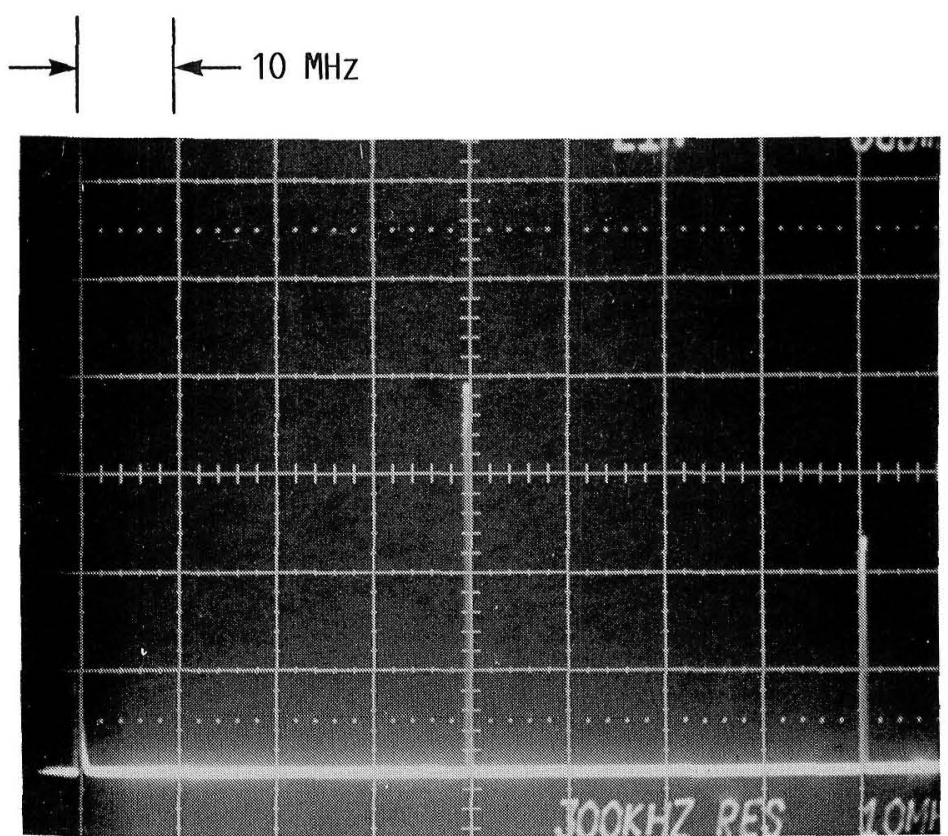

个 $\mathrm{Hz}$

(b)

FIGURE 2. - SIGNAL THAT REACHES PHOTODETECTOR AFTER EXITING LOOP (a) AND ITS SPECTRUM (b). 


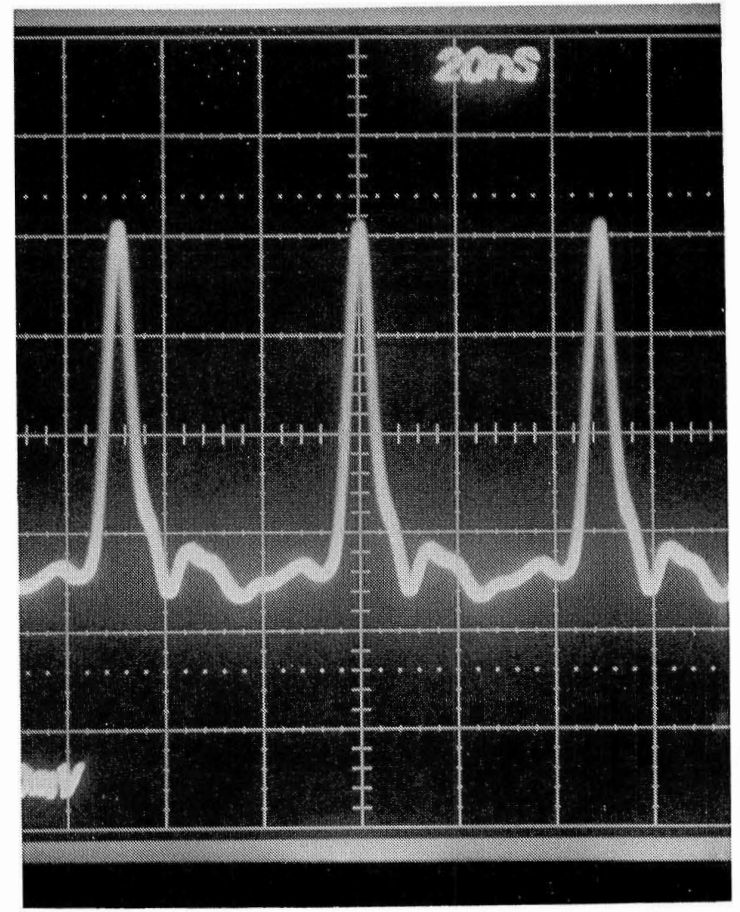

(a)

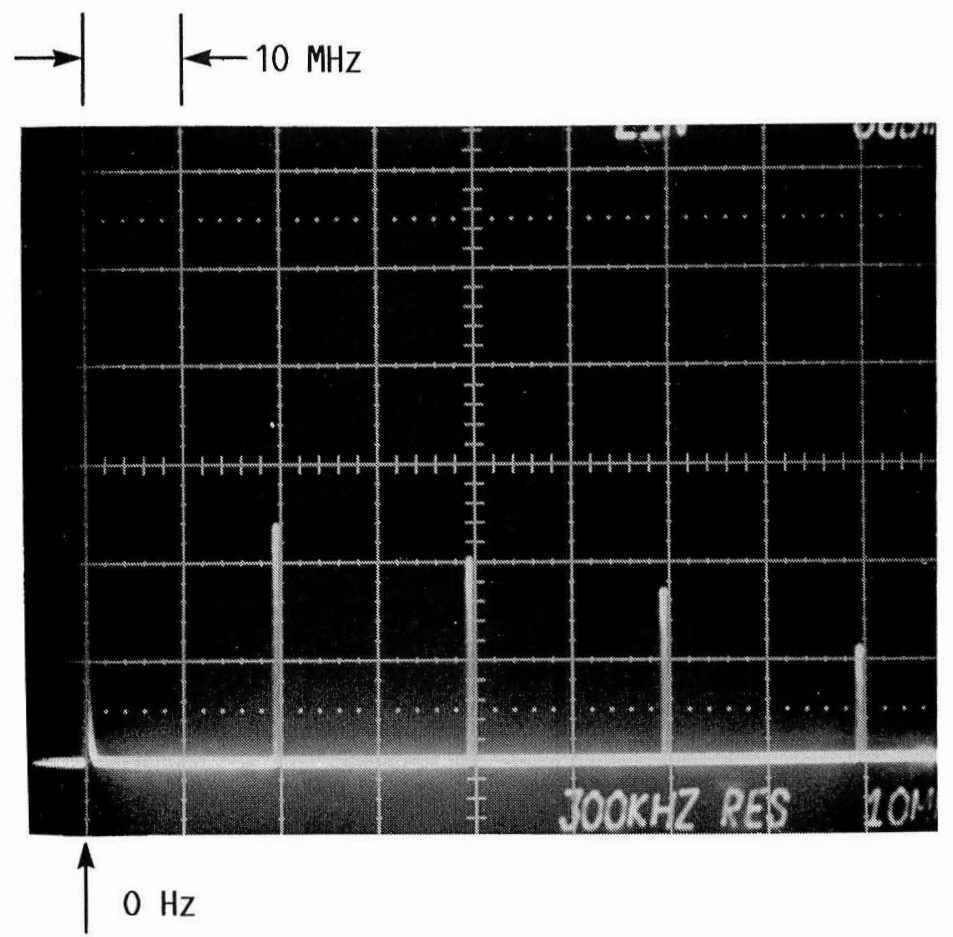

(b)

FIGURE 3. - SIGNAL THAT REACHES PHOTODETECTOR WHEN ENTRANCE TO LOOP IS BLOCKED (a) AND ITS SPECTRUM (b). 


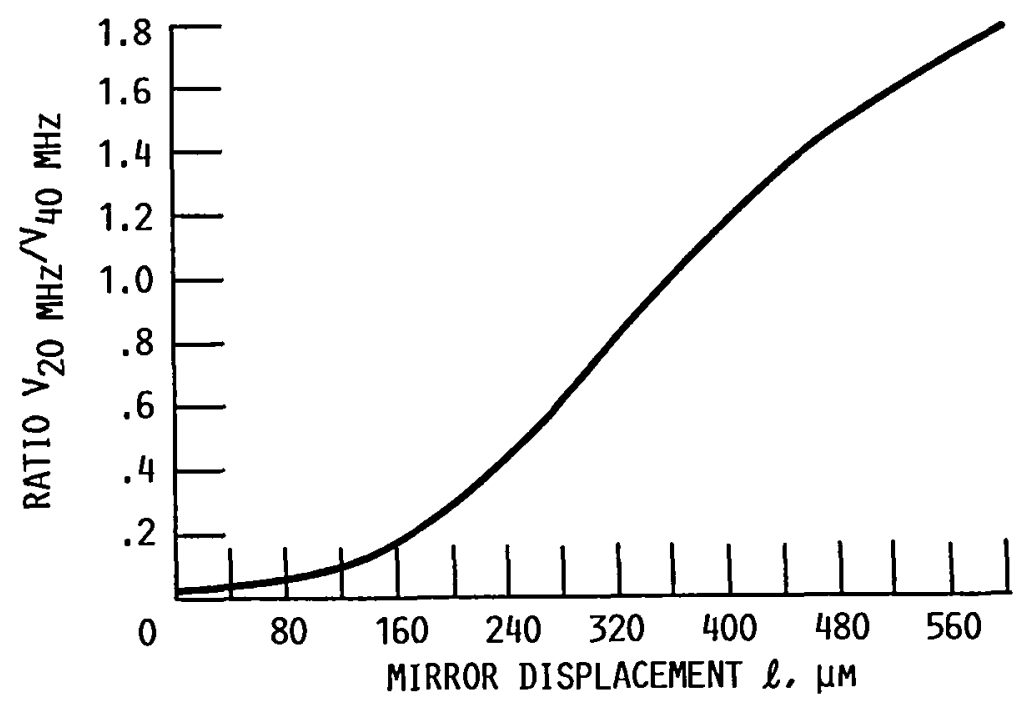

FIGURE 4.- EXPERIMENTALLY OBTAINED RELATIONSHIP BETWEEN THE RATIO $V_{20} \mathrm{MHz}_{2} / \mathrm{v}_{40} \mathrm{MHz}$ AND THE MIRROR $M_{2}$ DISPLACEMENT OVER THE RANGE FROM 0 TO $600 \mu \mathrm{M}$. 


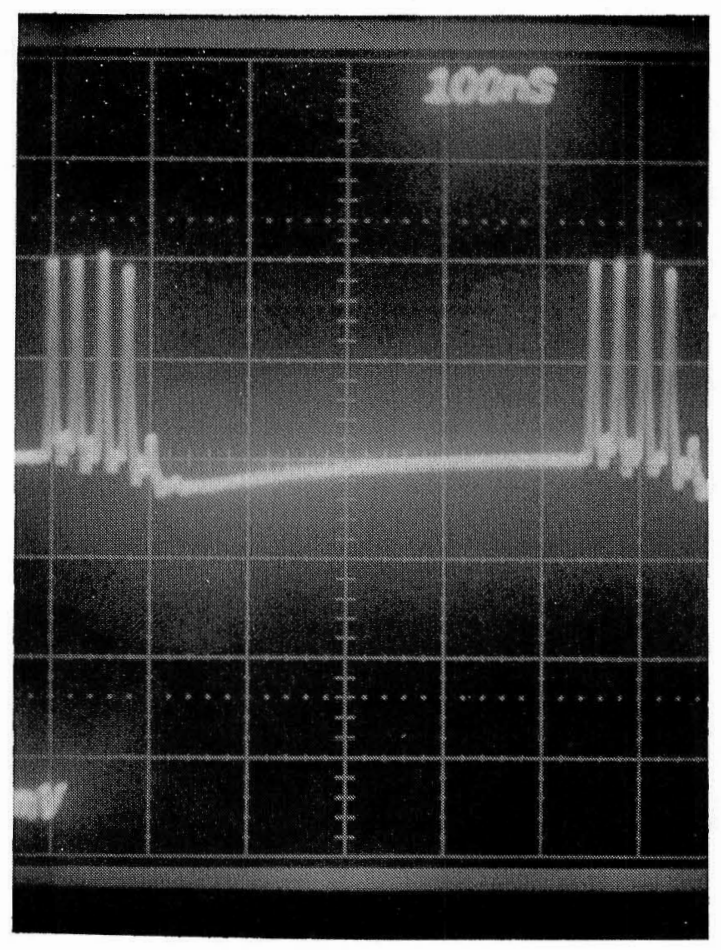

(a)
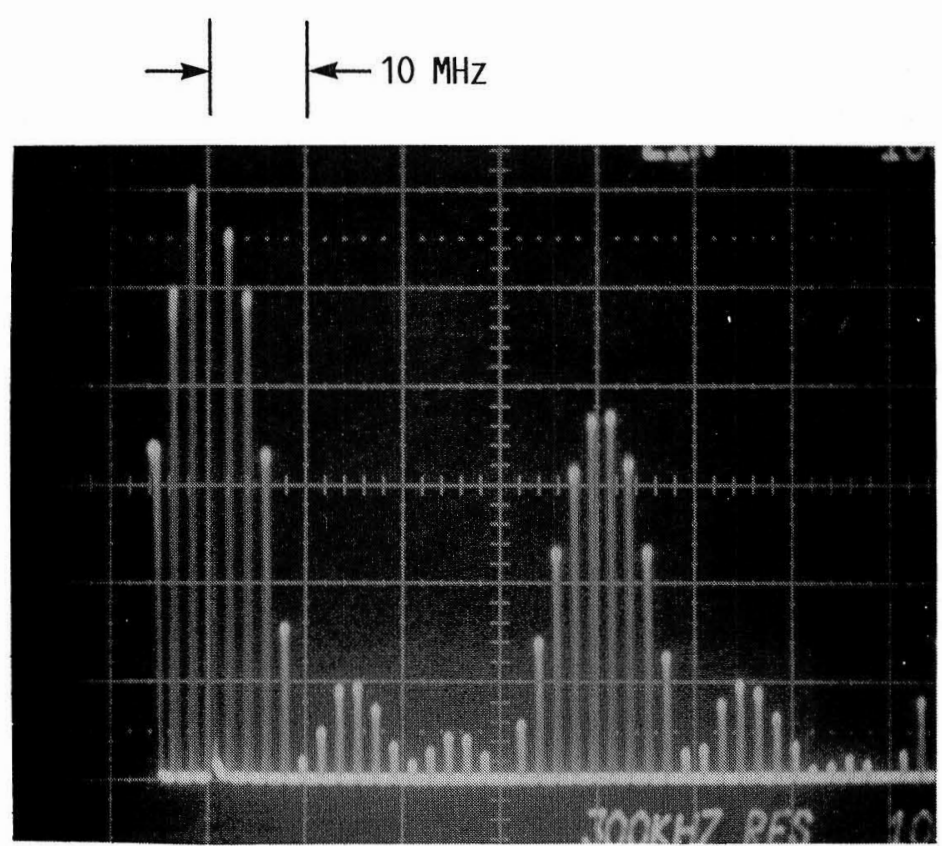

$\uparrow \mathrm{OHz}$

(b)

FIGURE 5. - SIGNAL THAT REACHES PHOTODETECTOR AFTER EXITING LOOP (a) AND ITS SPECTRUM ( $b$ ) IN SYSTEM WITH INITIAL DOUBLE PULSE. 


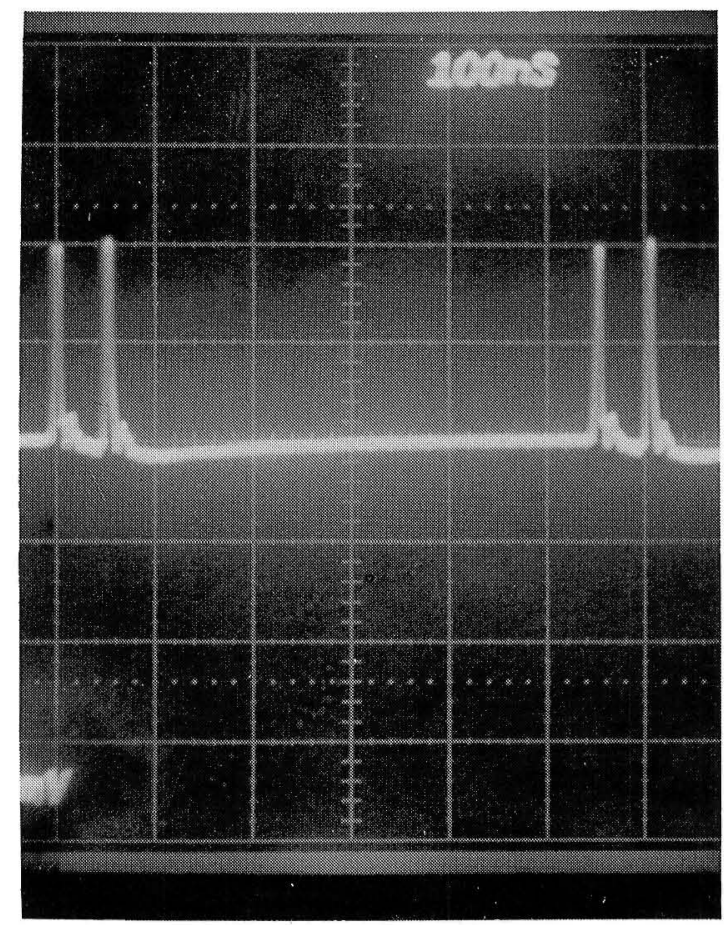

(a)
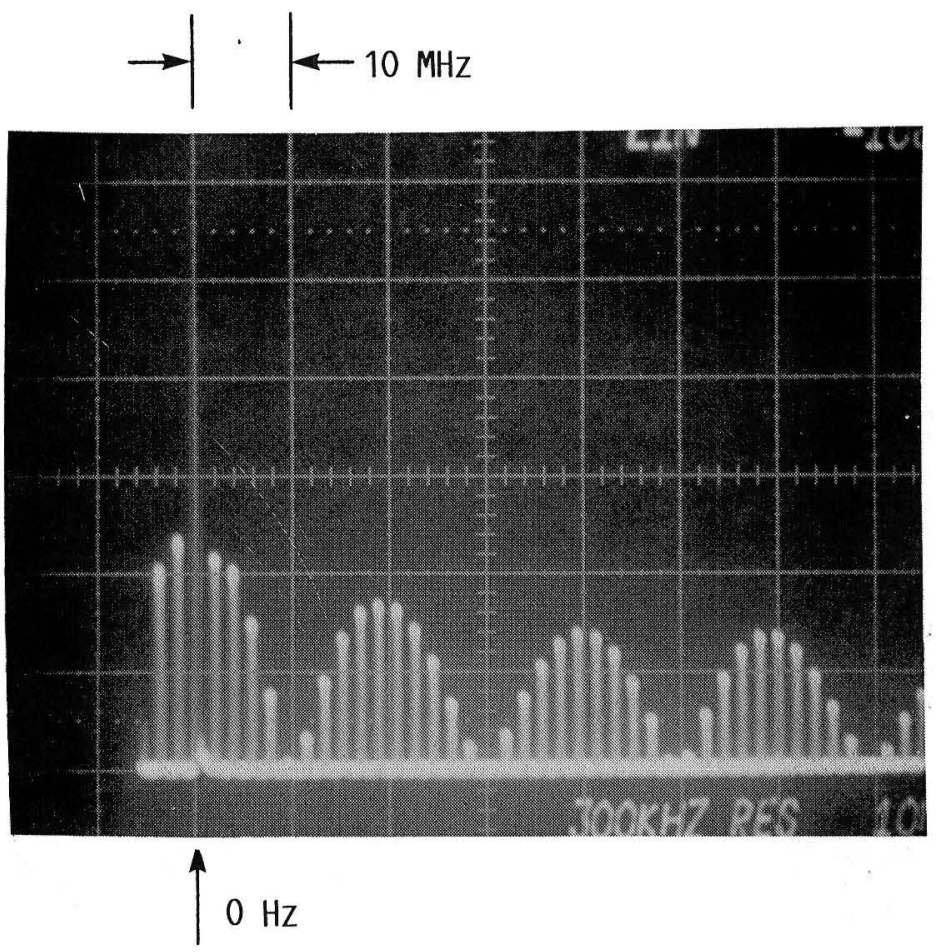

(b)

FIGURE 6. - SIGNAL THAT REACHES PHOTODETECTOR WHEN ENTRANCE TO LOOP IS BLOCKED (a) AND ITS SPECTRUM (b) IN SYSTEM WITH INITIAL DOUBLE PULSE. 


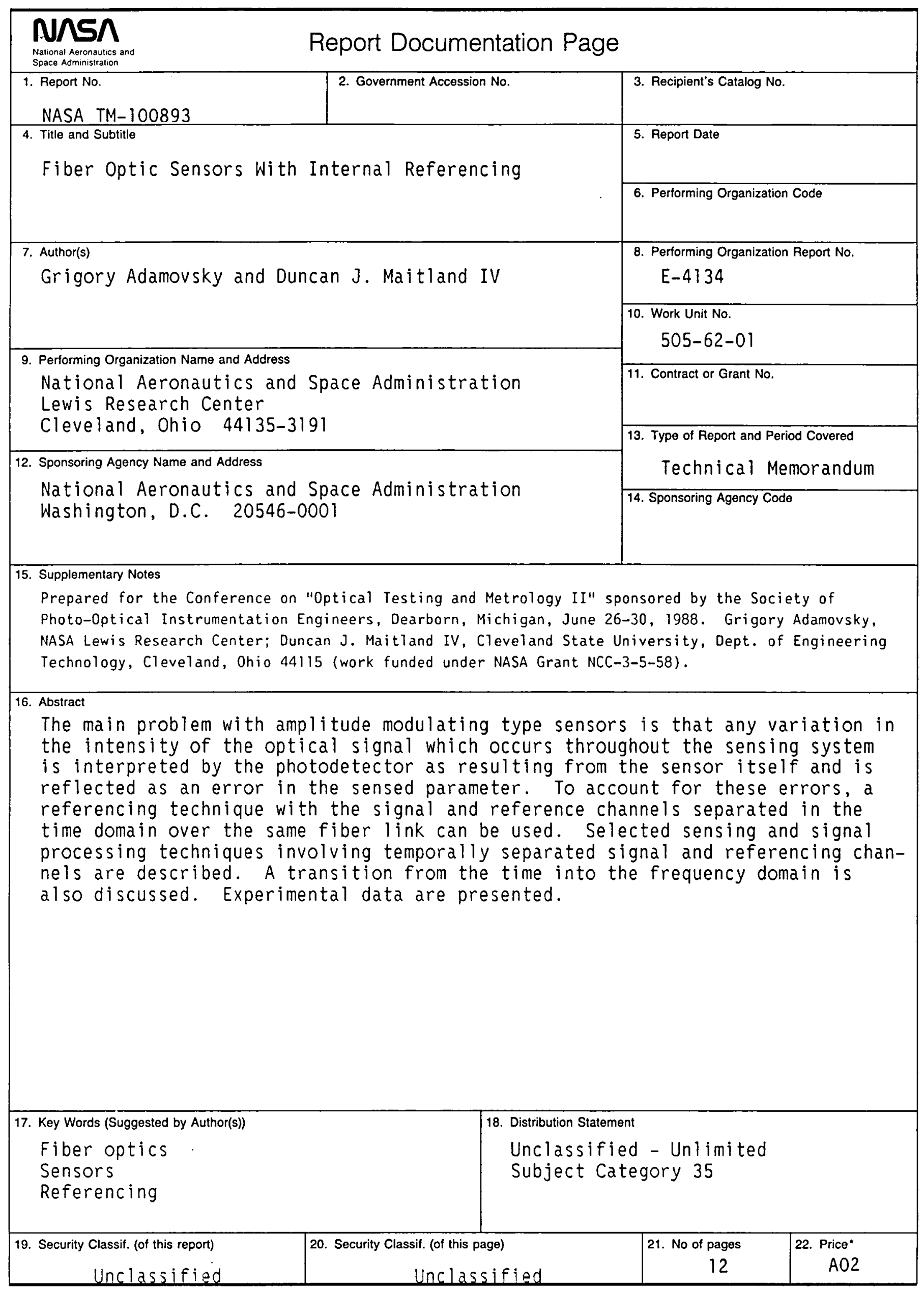


National Aeronautics and Space Administration

Lewis Research Center

Cleveland, Ohio 44135

Otficial Business

Penalty for Private Use $\mathbf{5 3 0 0}$
FOURTH CLASS MAIL

ADDRESS CORRECTION REQUESTED
|||||

H II LANGLEY RESEARCH CENTER

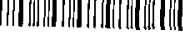

3 1176013267852

Postage and Fees Paid

National Aeronautics and

Space Administration

NASA 451 